\title{
INTERPOLATION FOR ENTIRE FUNCTIONS OF EXPONENTIAL TYPE AND A RELATED TRIGONOMETRIC MOMENT PROBLEM
}

\author{
ROBERT M. YOUNG
}

\begin{abstract}
A classical theorem of Hausdorff-Young shows that when $1<p<2$, the system of equations $\hat{\varphi}(n)=c_{n}(-\infty<n<\infty)$ admits a solution $\varphi$ in $L^{q}(-\pi, \pi)$ whenever $\left\{c_{n}\right\} \in l^{p}$. Here, as usual, $\hat{\varphi}$ denotes the complex Fourier transform of $\varphi$ and $q$ is the conjugate exponent given by $p^{-1}+q^{-1}=1$. The purpose of this note is to show that if a set $\left\{\lambda_{n}\right\}$ of real or complex numbers is "sufficiently close" to the integers, then the corresponding system $\hat{\varphi}\left(\lambda_{n}\right)=c_{n}$ is also solvable for $\varphi$ whenever $\left\{c_{n}\right\} \in l^{p}$. The proof is accomplished by establishing a similar interpolation theorem for a related class of entire functions of exponential type.
\end{abstract}

1. Introduction. A classical theorem of Hausdorff-Young shows that when $1<p<2$, the system of equations $\hat{\varphi}(n)=c_{n}(-\infty<n<\infty)$ admits a solution $\varphi$ in $L^{q}(-\pi, \pi)$ whenever $\left\{c_{n}\right\} \in l^{p}$. Here, as usual, $\hat{\varphi}$ denotes the complex Fourier transform of $\varphi$ and $q$ is the conjugate exponent given by $p^{-1}+q^{-1}=1$. In this note we show that if a set $\left\{\lambda_{n}\right\}$ of real or complex numbers is "sufficiently close" to the integers, then the corresponding system

$$
\hat{\varphi}\left(\lambda_{n}\right)=c_{n} \quad(-\infty<n<\infty)
$$

admits a solution $\varphi$ in $L^{q}(-\pi, \pi)$ whenever $\left\{c_{n}\right\} \in l^{p}$. Specifically, we have the following result.

THEOREM 1. Let $1<p<2$ and let $q$ be the conjugate exponent. There exists a constant $L>0$ with the following property: If $\left|\lambda_{n}-n\right| \leqslant L$, then the system (1) admits a solution $\varphi$ in $L^{q}(-\pi, \pi)$ whenever $\left\{c_{n}\right\} \in l^{p}$.

We prove Theorem 1 by establishing a similar interpolation theorem for a related class of entire functions of exponential type.

2. Interpolation in a related Banach space of entire functions. We denote by $E_{\tau}^{p}(p \geqslant 1)$ the Banach space of entire functions of exponential type $\tau$ for which

$$
\|f\|_{p}=\left\{\int_{-\infty}^{\infty}|f(x)|^{p} d x\right\}^{1 / p}<\infty .
$$

A sequence $\left\{\lambda_{n}\right\}$ of distinct real or complex numbers is said to be an interpolating sequence for $E_{\tau}^{p}$ if $T E_{\tau}^{p} \supset l^{p}$, where $T$ is given by $T f=\left\{f\left(\lambda_{n}\right)\right\}$. (Such sequences were studied extensively in [5] for the special cases $p=1,2$

Received by the editors April 14, 1975 and, in revised form, May 27, 1975.

AMS (MOS) subject classifications (1970). Primary 30A80; Secondary 42A80.

$\mathrm{Key}$ words and phrases. Interpolating sequence, entire functions of exponential type.

- American Mathematical Society 1976 
and the limiting case $p=\infty$. For general properties of the spaces $E_{\tau}^{p}$ see [2].) It is well known that $E_{\tau}^{p}$ is closed under differentiation and that

$$
\left\|f^{\prime}\right\|_{p} \leqslant \tau\|f\|_{p} .
$$

A simple application of the closed graph theorem shows that if $\left\{\lambda_{n}\right\}$ is an interpolating sequence, then the unit ball in $l^{p}$ can be interpolated in a uniformly bounded way [5]; that is, there exists a constant $M$ such that whenever $c \in l^{p},\|c\| \leqslant 1$, there corresponds at least one function $f$ in $E_{\tau}^{p}$ for which $T f=c$ and $\|f\| \leqslant M$. As a consequence [5], if the imaginary part of $\lambda_{n}$ is uniformly bounded, then the $\lambda_{n}$ are of necessity separated, that is $\inf \left|\lambda_{n}-\lambda_{m}\right|>0(n \neq m)$. But then, it follows that for every function $f$ in $E_{\tau}^{p}$,

$$
\left\{\sum_{n}\left|f\left(\lambda_{n}\right)\right|^{p}\right\}^{1 / p} \leqslant A\|f\|_{p}
$$

where $A=A\left(p, \tau,\left\{\lambda_{n}\right\}\right)$ is an absolute constant, independent of $f[6]$. Hence, in this case, $T$ is in fact a bounded linear transformation into $l^{p}$.

If $S: E_{\tau}^{p} \rightarrow l^{p}$ is defined by $S f=\left\{f\left(\mu_{n}\right)\right\}$, then we shall wish to conclude that $S E_{\tau}^{p}=l^{p}$ knowing that $T E_{\tau}^{p}=l^{p}$ and that $S$ is "close" to $T$. For this purpose, we will need the following interesting result of Bade and Curtis [1].

Lemma 1. Let $X$ and $Y$ be Banach spaces and $T: X \rightarrow Y$ a bounded linear transformation. Suppose that there exist constants $M>0$ and $0<\epsilon<1$ with the following property: For each $y$ in the unit ball of $Y$, there exists an $x$ in $X$ with $\|x\| \leqslant M$ and $\|T x-y\| \leqslant \epsilon$. Then $T$ is onto.

The proof of the following lemma is similar to that given in [3] for the case $p=2$.

LEMMA 2. Let $\left\{\lambda_{n}\right\}$ be a separated sequence of points lying in a strip parallel to the real axis, and suppose that $\left|\mu_{n}-\lambda_{n}\right| \leqslant L$. Then for every function $f$ belonging to $E_{\tau}^{p}$, we have the inequality

$$
\left\{\sum_{n}\left|f\left(\mu_{n}\right)-f\left(\lambda_{n}\right)\right|^{p}\right\}^{1 / p} \leqslant A\left(e^{\tau L}-1\right)\|f\|_{p}
$$

where $A$ is the same constant appearing in (3).

PRoof. Using Taylor's theorem, we write

$$
f\left(\mu_{n}\right)-f\left(\lambda_{n}\right)=\sum_{k=1}^{\infty} \frac{f^{(k)}\left(\lambda_{n}\right)}{k !}\left(\mu_{n}-\lambda_{n}\right)^{k} .
$$

Then, for any $\rho>0$,

$$
f\left(\mu_{n}\right)-f\left(\lambda_{n}\right)=\sum_{k=1}^{\infty} \frac{f^{(k)}\left(\lambda_{n}\right)}{\rho^{k}(k !)^{1 / p}} \cdot \frac{\rho^{k}\left(\mu_{n}-\lambda_{n}\right)^{k}}{(k !)^{1 / q}},
$$

and hence, by Hölder's inequality,

$$
\left|f\left(\mu_{n}\right)-f\left(\lambda_{n}\right)\right| \leqslant\left\{\sum_{k=1}^{\infty} \frac{\left|f^{(k)}\left(\lambda_{n}\right)\right|^{p}}{\rho^{k p} k !}\right\}^{1 / p} \cdot\left\{\sum_{k=1}^{\infty} \frac{(\rho L)^{k q}}{k !}\right\}^{1 / q} .
$$


Now, since $f^{(k)} \in E_{\tau}^{p}$, it follows from (2) and (3) that

$$
\sum_{n}\left|f^{(k)}\left(\lambda_{n}\right)\right|^{p} \leqslant A^{p} \tau^{k p}\|f\|_{p}^{p} .
$$

Therefore, we conclude that

$$
\begin{aligned}
\left\{\sum_{n}\left|f\left(\mu_{n}\right)-f\left(\lambda_{n}\right)\right|^{p}\right\}^{1 / p} & \leqslant A\|f\|_{p}\left\{\sum_{k=1}^{\infty} \frac{\tau^{k p}}{\rho^{k p} k !}\right\}^{1 / p}\left\{\sum_{k=1}^{\infty} \frac{(\rho L)^{q}}{k !}\right\}^{1 / q} \\
& =A\|f\|_{p}\left\{e^{\tau p \rho^{-p}}-1\right\}^{1 / p}\left\{e^{\rho^{q} L^{q}}-1\right\}^{1 / q}
\end{aligned}
$$

and the result follows by taking $\rho=\tau^{1 / q} L^{-1 / p}$.

The proof of Theorem 1 will follow easily from the following interpolation theorem for $E_{\tau}^{p}$.

THEOREM 2. Let $\left\{\lambda_{n}\right\}$ be a sequence of points lying in a strip parallel to the real axis. If $\left\{\lambda_{n}\right\}$ is an interpolating sequence for $E_{\tau}^{p}$, then there exists a constant $L>0$ such that $\left\{\mu_{n}\right\}$ is also an interpolating sequence for $E_{\tau}^{p}$ whenever $\left|\mu_{n}-\lambda_{n}\right| \leqslant L$.

Proof. Since $\left\{\lambda_{n}\right\}$ is interpolating for $E_{\tau}^{p}$, the unit ball of $l^{p}$ can be interpolated in a uniformly bounded way. Thus, there exists a constant $M$ such that whenever $\sum\left|c_{n}\right|^{p} \leqslant 1$, there exists a function $f$ in $E_{\tau}^{p}$ with $f\left(\lambda_{n}\right)=c_{n}$ and $\|f\|_{p} \leqslant M$.

Let us define a mapping $T: E_{\tau}^{p} \rightarrow l^{p}$ by $T f=\left\{f\left(\mu_{n}\right)\right\}$. The inequality (4) shows that $T$ is a bounded linear transformation into $l^{p}$. We show that $T$ is in fact onto $l^{p}$. Let $c=\left\{c_{n}\right\}$ belong to the unit ball of $l^{p}$ and choose $f$ in $E_{\tau}^{p}$ such that $\|f\|_{p} \leqslant M$ and $f\left(\lambda_{n}\right)=c_{n}$. Then (4) becomes

$$
\|T f-c\| \leqslant A M\left(e^{\tau L}-1\right),
$$

and since $L$ can be chosen small enough so that the right-hand side of (5) is less than 1 , the conclusion follows from Lemma 1.

Corollary. If $1<p<2$, then $\left\{\lambda_{n}\right\}$ is an interpolating sequence for $E_{\pi}^{p}$ whenever $\left|\lambda_{n}-n\right| \leqslant L$ and $L$ is sufficiently small.

Proof. In view of Theorem 2, it is enough to show that the integers are an interpolating sequence for $E_{\pi}^{p}$. Suppose that $\left\{c_{n}\right\} \in l^{p}$. By the HausdorffYoung theorem, there exists a function $\varphi$ in $L^{q}(-\pi, \pi)$ such that $\hat{\varphi}(n)$ $=c_{n}(-\infty<n<\infty)$. Since $\{\hat{\varphi}(n)\} \in l^{p}$ and $p>1$, it follows that $\hat{\varphi}(x)$ $\in L^{p}(-\infty, \infty)$ [4]. Thus, the function $\hat{\varphi}(z)$ belongs to $E_{\pi}^{p}$ and the proof is complete.

REMARK. For $p=1$, the integers fail to be an interpolating sequence for $E_{\pi}^{1}$ for the trivial reason that the nonzero integers are already a set of uniqueness. It was shown in [5], however, that $T E_{\tau}^{1}=l^{1}$ for every $\tau>\pi$.

3. Proof of Theorem 1. The proof of Theorem 1 follows immediately from the above corollary since every function $f$ belonging to $E_{\pi}^{p}$ is of the form $f=\hat{\varphi}$ for some $\varphi$ in $L^{q}(-\pi, \pi)[2]$. 


\section{REFERENCES}

1. W. G. Bade and P. C. Curtis, Jr., Embedding theorems for commutative Banach algebras, Pacific J. Math. 18 (1966), 391-409. MR 34 \# 1878.

2. R. P. Boas, Jr., Entire functions, Academic Press, New York, 1954. MR 16, 914.

3. R. J. Duffin and A. C. Schaeffer, A class of nonharmonic Fourier series, Trans. Amer. Math. Soc. 72 (1952), 341-366. MR 13, 839.

4. T. Kawata, A relation between the theories of Fourier series and Fourier transforms, Proc. Imp. Acad. Tokyo 16 (1940), 255-261. MR 2, 94.

5. R. M. Young, Interpolation in a classical Hilbert space of entire functions, Trans. Amer. Math. Soc. 192 (1974), 97-114.

6. —, An extension of the Hausdorff-Young theorem, Proc. Amer. Math. Soc. 45 (1974), $235-236$.

Department of Mathematics, Oberlin College, Oberlin, Ohio 44074 